\title{
MANTENIMIENTO DE LOS EFECTOS PRODUCIDOS POR LA REHABILITACIÓN PULMONAR EN PACIENTES CON EPOC: UNA REVISIÓN SISTEMÁTICA DE LITERATURA
}

\section{MAINTENANCE OF THE EFECTS PRODUCED BY LUNG REHABILITATION IN PATIENTS WITH COPD: A REWIEV LITERATURE ${ }^{1}$}

\author{
Miguel Avenza Jaén ${ }^{2}$ \\ Hospital Carlos III Madrid, España \\ miguelavenzajaen@gmail.com
}

\begin{abstract}
Resumen
La Enfermedad Pulmonar Obstructiva Crónica (EPOC) es un padecimiento prevenible y tratable, sin embargo, se ubica como la cuarta patología con más causas de muerte en el mundo. Considerando los diferentes manejos de esta enfermedad, el objetivo del presente artículo es identificar, a través de una revisión sistemática de literatura, patrones rehabilitadores correctos, con indicaciones concretas que ayuden a tratar esta patología con la máxima eficiencia y perdurabilidad en el tiempo. Para ello se consultan las bases de datos PudMed, Med Line y Embase entre los meses de diciembre de 2010 a diciembre de 2014. Los criterios de inclusión de artículos fueron todas las publicaciones randomizadas que mencionaran beneficios a corto o largo plazo de la rehabilitación pulmonar en una población con un diagnóstico de EPOC, determinada mediante exámenes físicos o por los actuales criterios de diagnóstico; se excluyeron otras condiciones pulmonares crónicas. Se concluye que, independientemente de la rehabilitación respiratoria brindada, se necesita un cambio en el estilo de vida del paciente para el mantenimiento de los efectos.
\end{abstract}

Palabras Claves: Ejercicio respiratorios, Efectos largo plazo, Tolerancia al ejercicio, Calidad de vida

\begin{abstract}
Chronic Obstructive Pulmonary Disease (COPD) is preventable and treatable, yet it is the fourth condition with most causes of global death. Considering different management of this disease, the objective of this article is identify, through a literature review, right rehabilitation patterns with specific indications to treat the diseases with maximum efficiency and durability over time. To do that, were done an online consult of databases in PudMed, Med and EMBASE, between december 2010 to December 2014. Inclusion criteria for articles were: benefits of a short or long-term rehabilitation, pulmonary scam COPD Diagnosis, through physical exams; other chronic lung conditions were excluded. We conclude that independence of respiratory rehabilitation provided, the patient needed a change in the lifestyle for maintenance of the effects
\end{abstract}

Key Words: Breathing Exercises, Long term Effects, Exercise tolerance, Quality of life.

\section{Introducción}

La enfermedad pulmonar obstructiva crónica (EPOC) es prevenible y tratable. Se caracteriza por la obstrucción progresiva y no completamente reversible del flujo aéreo, $\mathrm{y}$ 
se asocia con una respuesta inflamatoria pulmonar anómala a partículas o gases tóxicos, fundamentalmente el humo del tabaco (GOLD, 2006).

La obstrucción al flujo aéreo se define por la espirometría cuando el cociente FEV1/FVC post-broncodilatadores es menor de 0,7 (o por debajo del límite inferior de la normalidad en sujetos mayores de 60 años) (SEPAR/ ALLAT-ULASTER, 2009). Actualmente, se reconoce la afectación sistémica extra-pulmonar que puede contribuir a la gravedad en algunos pacientes.

La prevalencia mundial de la EPOC oscila entre el 5 y el $10 \%$, ha aumentado en las últimas décadas y es más frecuente en hombres que en mujeres dada la mayor prevalencia de tabaquismo en los hombres, aunque esto se espera que cambie en las próximas décadas ya que el consumo de tabaco en mujeres jóvenes es significativamente mayor al de los hombres jóvenes (Pride, 1990). En España se han realizado varios estudios epidemiológicos con base poblacional que han dado como resultado una prevalencia global de 9,1 \% en edades comprendidas entre los 40 y los 70 años (Brotons, Pérez, Sánchez-Toril, Soriano, Hernández y Belenguer, 1999; Peña, Miravitlles, Gabriel, Jiménez-Ruiz, Villasante y Masa, 2000 y Sobradillo, Miravitlles, Jiménez, Gabriel, Viejo y Masa, 1999).

La EPOC está fundamentalmente asociada al tabaquismo, sobre todo en países desarrollados, aunque en algunas sociedades menos desarrolladas también puede asociarse a la exposición a humos tóxicos como la combustión de leña. La mortalidad de la EPOC la ubica en la cuarta posición a nivel global, con 3 millones de muertes y un porcentaje de un 5,1\% en el año 2004, sin embargo, se prevé un ascenso de esta tendencia que podría ubicarla como la tercera causa de muerte para el 2020 (WHO, 2008) $)^{[]}$.

El tratamiento consiste principalmente en dos grandes pilares. En primer lugar, se encuentra la prevención orientada al abandono de hábitos tabáquicos, que constituye una de las intervenciones más importantes para evitar el deterioro funcional del paciente con EPOC, y la realización de ejercicio físico regular, recomendable en todos los estadios de la enfermedad (SEPAR/ ALLAT-ULASTER, 2009). En segundo lugar, se encuentra el tratamiento médico, que está compuesto por la vacunación antigripal y neumocócica, los broncodilatadores, que son los más eficaces a la hora de reducir los síntomas de los pacientes con EPOC, no sólo por el ensanchamiento de la luz bronquial sino porque pueden ayudar a eliminar secreciones o mejorar, incluso, la contractilidad del diafragma. El control del estado nutricional y mental junto a la valoración de contar o no con la oxigenoterapia domiciliaria en pacientes graves $(\mathrm{PaO} 2<55$ $\mathrm{mmHg}$ o $\mathrm{SaO} 2<88 \%$ ) será el último control para completar el tratamiento médico.

\section{Propósito}

La revisión sistemática de bibliografía agrupó todos aquellos estudios randomizados que indicaran un tratamiento de la rehabilitación pulmonar en los pacientes con EPOC, diagnosticados mediante exámenes físicos o por los actuales criterios, a fin de evaluar sus beneficios en el corto o largo plazo. De esta forma, en una población de personas con un diagnóstico de EPOC, el 
objetivo de la revisión fue obtener un patrón rehabilitador correcto, con indicaciones concretas que ayuden a tratar una patología que actualmente es la cuarta causa de muerte en el mundo. Lo anterior se propuso para facilitar la búsqueda de estrategias que ahorren y no eleven el gasto sanitario para que finalmente los pacientes tengan un beneficio a largo plazo, con una mejor calidad de vida relacionada con la salud (CVRS).

\section{Metodología}

La búsqueda literaria se realizó a través de las bases de datos PudMed, Med Line y Embase de diciembre del 2010 a diciembre del 2014 con limitación de textos en inglés y español. Los términos que se utilizaron en la búsqueda fueron los siguientes: pulmonary rehab, exercise training, chronic obstructive pulmonary disease (COPD), effects, exercise tolerance, health reated quality of life, randomized controlled trial, clinical trial, meta-analisis, review, practice guidelines. Posteriormente, se realiza una búsqueda avanzada con los términos: "Pulmonary rehabilitation and COPD and quality of life and excersise". Los criterios de inclusión fueron todo estudio o publicación randomizado que hablara sobre los beneficios a corto o largo plazo en una población de personas con un diagnóstico de EPOC determinada, mediante exámenes físicos o por los actuales criterios de diagnóstico, en función de las características del programa rehabilitador, sus efectos, la duración de los efectos del programa rehabilitador, las estrategias para poder mantenerlos durante el mayor tiempo posible para mejorar la tolerancia al ejercicio, la disnea, la calidad de vida y reducir las recaídas y el costo médico sanitario. Se excluyeron otras condiciones pulmonares crónicas

Sobre el proceso de clasificación, de un examen inicial se obtuvieron 181 artículos. De este total, se eliminan 102 artículos bajo un criterio de exclusión de aquellos que no evaluaban el tiempo de duración en los efectos de entrenamiento o que no abordaban ningún tema del efecto relacionado con su duración como lo son cuestiones de durabilidad, fuerza y masa muscular, técnicas para mejorar los efectos e incluso el estudio de estos.

Los artículos fueron clasificados en una escala del 1 al 3. Se toma una puntuación con tres valores para dividir los 79 artículos seleccionados en función del peso informativo referente a la durabilidad de los efectos conseguidos con el entrenamiento. Se valora con una puntuación de 1 aquellos artículos que tenían muy poco peso o valor informativo, con un 2 los artículos con un peso intermedio en la investigación de los efectos y con un 3 aquellos con un alto valor informativo. De los 79 artículos seleccionados, un total de 33 artículos (47,1\%) tuvo una puntuación de 1; 32 artículos (40,5\%), una puntuación de 2 y 14 artículos (17,7\%), una puntuación 3.

\section{Marco teórico}

Los programas de rehabilitación para pacientes con EPOC están bien establecidos como medio de la mejora de la terapia estándar, a fin de controlar y aliviar los síntomas y la capacidad funcional óptima (Gold y Shadlen, 2007, Ries et al., 2007). Su objetivo principal es restablecer al 
paciente al nivel más alto posible de la función independiente, que sean más activos físicamente y menos dependientes a los profesionales de la salud y los recursos médicos.

La intolerancia al ejercicio es el principal factor que limita la participación en las actividades de la vida diaria en personas con EPOC. Los síntomas en la mayoría de los pacientes son la disnea y la fatiga -consecuencias de las limitaciones ventilatorias-, el intercambio pulmonar de gases, la disfunción muscular periférica y la disfunción cardiaca. La ansiedad, la depresión y la falta de motivación también se asocian con intolerancia al ejercicio.

La rehabilitación pulmonar puede ser evaluada desde tres perspectivas diferentes: el paciente, el programa y la sociedad (Nici et al., 2006). Se trata de un servicio que cumple con la definición general de la rehabilitación y logra su terapéutica con objetivos implementados a través de la construcción de programas, que dependen tanto de las culturas como de los sistemas de salud. No obstante, no hay a nivel internacional una fórmula reconocida para el diseño de un programa, por lo que puede haber diferencias significativas en el enfoque en función de un individuo o población. En algunos países, el proceso se centra en el individuo y en el óptimo beneficio para el paciente. En otros países donde los recursos son limitados, los programas son configurados para proporcionar el máximo beneficio para la población utilizando al mínimo la rehabilitación necesaria para obtener un resultado satisfactorio. Sea cual sea la estrategia, el programa debe ser estructurado de una manera que incluya a los pacientes y la familia, y resulte en un cambio de estilo de vida que pueda sostener las mejoras durante el mayor tiempo posible.

La rehabilitación pulmonar se debe considerar para todos los pacientes con enfermedad respiratoria crónica que presentan síntomas persistentes, limitada actividad física o son incapaces de adaptarse a la enfermedad a pesar del óptimo tratamiento médico. Los cambios de hábitos y de estilo de vida, especialmente el mantenimiento regular de ejercicio, son muy importantes en la rehabilitación pulmonar y probablemente contribuyen a la disminución de los beneficios después de 18 a 24 meses.

La mayoría de la literatura reseñada se relaciona con programas de rehabilitación realizados durante un periodo de estabilidad clínica. Sin embargo, en los últimos años se han comenzado a publicar estudios en rehabilitación indicados después de una exacerbación, los cuales han demostrado efectos beneficiosos (Puhan, Scharplatz, Troosters y Steurer, 2005). De hecho, la rehabilitación para pacientes hospitalizados puede ofrecer beneficios similares a los pacientes ambulatorios (Goldstein, Gort, Stubbing, Avendaño y Guyatt, 1994).

En los primeros estudios reseñados, se tiene la desventaja del alto costo y la falta de cobertura por el seguro de algunos países, mientras que como ventajas se dispone de un entorno clínico seguro y la disponibilidad de personal capacitado. La rehabilitación ambulatoria es la más ampliamente disponible. En mayor cuantía, los estudios describen los beneficios de la rehabilitación pulmonar derivada de los programas de pacientes externos del hospital, con rehabilitación a domicilio, lo cual ofrece mayor comodidad para el paciente y prolonga su beneficio (Goldstein et al., 1994 y Strijbos, Postma, Van Altena, Gimeno y Koeter, 1996). 
No obstante, en pacientes gravemente discapacitados, la rehabilitación en casa puede no ser tan eficaz (Wedzicha, Bestall, Garrod, Garnham, Paul y Jones, 1998). Entre las desventajas citadas se encuentra la falta de oportunidad para los grupos, la limitada presencia de un equipo multidisciplinar, la disponibilidad variable de ejercicio, la falta de instalaciones seguras y el costo de las visitas por el profesional de la salud.

Aunque la rehabilitación pulmonar es segura, se recomienda que el personal tenga una formación en técnicas de reanimación. La rehabilitación pulmonar se inicia con la evaluación del paciente, seguido por el programa de rehabilitación pulmonar formal y el desarrollo de estrategias para mantener los beneficios a través de cambios de estilo de vida. Aunque los beneficios a corto plazo se pueden obtener incluso de los programas de hospitalización breve de 2 semanas, la rehabilitación a largo plazo consiste en programas generalmente de 8 a 12 semanas de duración, dependiendo de las características de cada paciente y la respuesta al tratamiento.

Los objetivos inmediatos de la rehabilitación pulmonar son reducir síntomas, mejorar la función física y mejorar la CVRS. Los objetivos a largo plazo son mantener estos beneficios y verlos traducido en la utilización de recursos reducidos de atención de salud, especialmente a través de la prevención de ingreso al hospital, la reducción de la duración de estancia hospitalaria y la mejora de la gestión propia, lo que limita la dependencia en la atención médica.

En términos generales, el tiempo de duración del beneficio de la rehabilitación pulmonar se refiere a la duración de los efectos beneficiosos de estos programas en la reducción de síntomas (disnea), la mejoría de la CVRS, la tolerancia al ejercicio y la incidencia de exacerbaciones futuras. Los estudios limitados sugieren que los beneficios de la rehabilitación pulmonar a sus valores basales van disminuyendo después de 6 a 12 meses, pero sigue mejorado en comparación con los sujetos control después de 1 año (Ries, Kaplan, Limberg y Prewitt, 1995 y Cambach, Wagenaar, Koelman, van Keimpema y Kemper, 1999). Los beneficios en la CVRS parecen ser mejores conservando el rendimiento del ejercicio, a veces sosteniéndola 2 años después de la intervención (Troosters, Gosselink y Decramer, 2000; Guell, Casan, Belda, Sangenis, Morante, Guyatt y Sanchis, 2000, y Foglio, Bianchi, Bruletti, Battista, Pagani y Ambrosino, 1999). En algunos estudios, estos beneficios parecen mantenerse en la ausencia de una determinada terapia de entrenamiento, lo que sugiere que un cambio en el estilo de vida altera el comportamiento.

\section{Resultados}

Actualmente, la rehabilitación pulmonar incluye varios componentes como el entrenamiento físico, la educación, la instrucción en diversas técnicas de fisioterapia y psicosociales. En los ensayos clínicos revisados y publicados desde 1990, se apoyan por completo los efectos beneficiosos de los programas de rehabilitación pulmonar.

El ejercicio físico considerado como la piedra angular de la rehabilitación pulmonar es el mejor medio disponible para mejorar la función muscular en el EPOC. En personas con EPOC,

Revista MHSalud@ (ISSN: 1659-097X) Vol. 12. No. 1. Febrero-Agosto, 2015 
se han reducido la tolerancia al ejercicio, la disnea de esfuerzo o fatiga, y el deterioro de las actividades de la vida diaria.

Las exacerbaciones agudas son excelentes candidatos para entrenamiento. Los programas de ejercicio de entrenamiento deben abordar al individuo, incluyendo la ventilación, el intercambio gaseoso y el trabajo de la musculación respiratoria. El ejercicio físico también puede mejorar la motivación para hacer ejercicio, reducir las perturbaciones del estado de ánimo, disminuir los síntomas y mejorar la función cardiovascular.

Una guía práctica del entrenamiento proporciona un mínimo de 20 sesiones tres veces por semana para lograr beneficios fisiológicos. También puede ser aceptado el entrenamiento dos veces por semana de forma supervisada y una sesión en casa sin supervisar. El ejercicio de alta intensidad produce mayores beneficios fisiológicos. Sin embargo, el entrenamiento de baja intensidad también es eficaz para aquellos pacientes que no pueden lograr este nivel de intensidad.

El entrenamiento interválico puede ser útil en los pacientes más sintomáticos. Tanto los ejercicios de las extremidades superiores e inferiores deben ser utilizados. Al respecto, la combinación del entrenamiento de resistencia y fuerza tiene múltiples efectos beneficiosos y es bien tolerado.

En un programa de entrenamiento, la deambulación se aconseja como un componente casi imprescindible de la rehabilitación, mejorando la disnea y la tolerancia al ejercicio que prácticamente son dos síntomas que van sujetos de la mano. Heppner (2006) propone un programa rehabilitador de 8 semanas de ejercicios presenciales y 12 semanas de mantenimiento, a base de marcha y ejercicios respiratorios. Las revisiones semestrales muestran la mejora en la disnea y la tolerancia al ejercicio, incluso, como en cualquier programa, aporta mejoría en la calidad de vida.

Se debe destacar que no solo la deambulación ha servido como método de entrenamiento aeróbico, sino que también el tapiz rodante o el cicloergómetro son utilizados en los distintos ensayos. Prácticamente el $100 \%$ de los artículos revisados han demostrado que la tolerancia al ejercicio ha mejorado o, en muy pocos casos, ha sido insignificante en la práctica de un entrenamiento pulmonar. En los ensayos valoramos esa mejora por la prueba de 6 minutos caminando 6MWT.

La disnea mejora mucho con la rehabilitación y va íntimamente relacionada con la capacidad de realizar ejercicio. Casaburi (2005) lo demuestra con un programa de ejercicios de 8 semanas con 3 sesiones semanales y la combinación de "tiotropio", el cual se mantiene durante 3 meses. Este se valora por distintas escalas como la Percepción de la disnea (POD), por las pruebas de transporte a pie y el Chronic Respiratory Disease Questionnaire (CRDQ), la más utilizada en algunos ensayos por la EVA. A su vez, Karapolat (2007) utiliza la escala EVA para valorar disnea, aunque típicamente se utiliza para valorar la fatiga muscular. Barakat, Michele, George, Nicole y Guy (2008) emplean la escala de Borg como instrumento más apropiado para abordar la fatiga cardiopulmonar. 
La rehabilitación pulmonar también va a incidir sobre la mejora de la CVRS. Múltiples estudios demuestran que con el entrenamiento pulmonar mejora la calidad de vida, mejorando sus actividades de la vida diaria. Finnerty, Keeping, Bullough y Jones (2001) proponen un programa rehabilitador ambulatorio de 6 semanas de duración, con 10 minutos (min) de calentamiento, 25 min de ejercicio aeróbico y 10 min de enfriamiento en el ambulatorio (1h por semana) y 5 veces por semana 10 min de marcha dos veces al día, el cual produce una mejora de la CVRS más allá de los 6 meses del programa. EI mantenimiento de la mejoría de la CVRS puede llegar a los 6 meses. En mayor parte, la CVRS se mide con escalas como el cuestionario respiratorio de St George's (SGRQ).

Otro efecto importantísimo de estudiar son las exacerbaciones, pues si se reducen las recaídas o, en su defecto, el número de hospitalizaciones y el número de días de hospitalización para los pacientes con EPOC, se reduce el coste médico-sanitario del sistema sanitario (en llamadas, visitas e ingresos). El grupo de rehabilitación pulmonar en colaboración con el grupo de rehabilitación cardiopulmonar de California afirma que 3 meses de rehabilitación pulmonar son eficaces para mejorar los síntomas y reducen la utilización de los recursos (visitas y llamadas médicas) más de 18 meses, los beneficios disminuyen paulatinamente, aunque los niveles se mantienen siempre por encima de los valores basales. Boxall, Barclay, Sayers y Caplan (2005) proponen su programa de caminar dividido en 10 niveles, ejercicios de miembros superiores (MMSS) en 18 niveles y 6 sesiones de educación durante 12 semanas. A una sesión semanal resulta un menor nivel de hospitalización y exacerbaciones en los 6 meses posteriores (California Pulmonary Rehabilitation Collaborative Group, 2005 y Boxall et al., 2005).

La depresión y la ansiedad son complicaciones de la enfermedad. Con el entrenamiento o programa de rehabilitación se consigue que estas complicaciones no aparezcan ya que el ejercicio y sus efectos producen una mejor calidad de vida, por tanto, mejoran las actividades de la vida diaria y con ello la mínima dependencia del paciente. Toshima, Kaplan y Ries (1990) demuestran beneficios a corto plazo (6 meses) e importantes beneficios fisiológicos y psicológicos con un programa muy completo que consta de 2 clases de educación semanales, terapia o entrenamiento físico supervisado, instrucciones respiratorias y apoyo psicosocial. La ansiedad está íntimamente ligada con la disnea.

La rehabilitación pulmonar es costo-efectiva en los pacientes. Actualmente existen pocos estudios suficientes para determinar la mejora del entrenamiento pulmonar frente al gasto del sistema sanitario en pacientes con EPOC realizando el tratamiento rehabilitador. Pero con la reducción de las exacerbaciones y el número de ingresos, llamadas o visitas médico-sanitarias, unido a la mejora de los efectos del entrenamiento, está ampliamente justificada esta práctica.

\section{Beneficios a corto y largo plazo: durabilidad de los efectos}

De acuerdo con la revisión literaria, la durabilidad de permanencia de los efectos deseados del programa rehabilitador va íntimamente ligado al tipo de entrenamiento o diseño del ejercicio o 
programa. Aquellos programas rehabilitadores que insisten más en el entrenamiento supervisado en la clínica o en el hogar y una buena educación y concienciación para una rutina de ejercicio a la vuelta a casa dan como resultado una mayor supervivencia de los efectos conseguidos. Ries, Kaplan, Myers, Prewitt y Lung (2003) plantean un ensayo con 36 pacientes distribuidos en tres grupos, llevando un entrenamiento pautado y guiado en clínica con 2 sesiones por semana durante un año, con lo que consiguen una disminución de los efectos a lo largo de 18 meses hasta los 24 meses. En cambio, aquellos programas que se preocupan menos de la educación y concienciación, y por lo tanto el ejercicio no supervisado en el hogar, consiguen mejoras pero menos duraderas a lo largo del tiempo. Pitta, Troosters, Probst, Langer, Decramer y Gosselink (2008) plantean un ensayo con 29 pacientes, de los cuales solo 12 finalizaron y el $41 \%$ fue hospitalizado por exacerbaciones. El entrenamiento tenía una duración de 6 meses y consistía en lo siguiente: durante los primeros 3 meses tuvieron 3 sesiones por semana y en los últimos 3 meses 2 sesiones por semana. Las mejoras perduraron 3 meses después del programa.

Por lo evaluado en los distintos artículos, no es tan importante el tipo de entrenamiento pero sí su supervisión. Existen entrenamientos muy completos que trabajan el ejercicio aeróbico, generalmente en bicicleta estática o en cinta. En esta línea, la propuesta de Spencer, Alison y McKeough (2010) contempla que durante 8 semanas y 5 días por semana, se expone a los pacientes de EPOC a un entrenamiento completo con tapiz rodante, bici, pedaleo de brazos, fuerza de MMSS y MMII, combinado con tratamiento en el hogar y técnicas de relajación, el cual produce un mantenimiento de los efectos de 12 meses. Mientras que Regiane, Gorostiza, Gáldiz, López de Santa María, Casan y Güell (2007), con otro entrenamiento supervisado, trabajo de educación, fisioterapia respiratoria y entrenamiento muscular, consiguen un mantenimiento de los efectos de 6 meses con un programa de 9 semanas y 1 sesión semanal (Ibíd. y Regiane, 2007). Pero incluso, los entrenamientos con menos técnicas a la hora del ejercicio son igual de efectivos en la durabilidad de sus efectos. Behnke, Jörres, Kirsten, Magnussen y Monaldi (2003) proponen un entrenamiento no tan preparado, con un programa combinado de tratamiento hospitalario, 10 sesiones supervisadas y en el hogar, enseñándoles hábitos de 15 min de caminata 3 veces por día, el cual perdura 18 meses.

El componente formal de la mayoría de los programas de rehabilitación pulmonar tiene una duración relativamente corta, por lo general entre 6 y 12 semanas. Varios ensayos clínicos de 6-12 semanas de un programa completo de rehabilitación pulmonar han demostrado que los beneficios en los pacientes persisten normalmente durante 12 a 18 meses después de la intervención, y poco a poco disminuyen a partir de ahí. Güell et al. (2000) afirman que esa persistencia puede incluso llegar a los 2 años.

Distintas evaluaciones indican que a mayor intensidad y cuanto más prolongados son los programas, más duración parecen tener de los efectos. Griffiths et al. (2000) crean un programa prolongado y de gran intensidad con resultados de 1 año de mantenimiento de los efectos. Este consta de 6 semanas de entrenamiento con 3 sesiones por semana hasta completar un total de 18 visitas de 2 horas cada una. El programa incluye actividad educativa, fisioterapia respiratoria, 
control nutricional, 30 min de ejercicios en MMSS y MMII, bicicleta estática y cinta a un 80 $\%$ de la FCmáx. Se prescribe oxígeno $\left(\mathrm{O}_{2}\right)$ suplementario en el ejercicio al que lo necesite. Finalmente, alientan a los pacientes a realizar el ejercicio en casa.

Sobre la durabilidad de los efectos, simplemente un cambio de modo de vida, de hábitos hacia el ejercicio y la actividad mejoran los efectos pero lógicamente la durabilidad será relativa en función de la intensidad, duración y conocimiento de la enfermedad. Así que los tres grandes parámetros que por los estudios presentes van a determinar la durabilidad de los efectos conseguidos con un entrenamiento o programa rehabilitador serán: la intensidad del ejercicio aeróbico, la duración del programa y el nivel de seguimiento o conocimiento y la educación de la enfermedad.

Tanto la baja intensidad del programa pulmonar como el ejercicio de alta intensidad producen beneficios clínicos en los pacientes de EPOC. Aunque el ejercicio de entrenamiento de mayor intensidad produce mayor beneficios fisiológicos que el entrenamiento de baja intensidad. El impacto de la intensidad del ejercicio en el resultado del mantenimiento de entrenamiento no ha sido evaluado.

La duración del programa también tiene una relación muy directa con el tiempo de duración de los efectos. Como se comentó anteriormente, un mayor periodo de programa de entrenamiento produce una mejoría en el mantenimiento de los efectos. Wijkstra et al. (1995) ensayaron con tres grupos a los que se aportaba 3 meses de entrenamiento con 2 sesiones a la semana en uno de los grupos, una sesión al mes para otro y el último grupo sin fisioterapia rehabilitadora. Todos ellos cuentan con sesiones educativas manteniendo el primero de los grupos los efectos a lo largo de los 18 meses. Programas de tres meses aseguran un larga permanencia de los efectos pero de menos de 6 semanas de entrenamiento hay pocos estudios que indiquen la supervivencia de los efectos, aunque los resultados dependerán de la intensidad del ejercicio. Programas mayores de 3 meses de duración no nos aseguran mayor durabilidad de los efectos. Güell et al. (2006) proponen un programa de 16 semanas con número de sesiones semanales progresivas de 2 a 5 a lo largo del programa pero no nos indican una durabilidad superior al de otro programa similar de menor duración, por tanto, sería más recomendable realizar recordatorios o visitas supervisadas para ampliar la supervivencia de los efectos.

El último aspecto a tomar en cuenta en la durabilidad de los efectos es el nivel de seguimiento o conocimiento y educación de la enfermedad. El paciente que conoce y se le educa en su enfermedad tomará más medidas para su cuidado y bienestar, lo que proporcionará una ayuda extra a la permanencia de los efectos. Boxall et al. (2005) y Engström, Persson, Larsson, Sullivan y Scand (1999) apoyan la hipótesis de que la duración de los efectos sobre la tolerancia al ejercicio se puede alcanzar después de 12 meses, en los que se incluya un periodo intensivo de tratamiento de pacientes ambulatorios, complementado con sesiones de refuerzo y un plan individual de supervisión y formación en casa. Este es el último factor que proporciona una garantía de mayor permanencia de los efectos conseguidos con un programa de rehabilitación pulmonar.

Finalmente, a continuación se exponen los artículos con mayor peso informativo referentes a la persistencia de los efectos conseguidos con un entrenamiento rehabilitador pulmonar. 
URL: http://www.revistas.una.ac.cr/mhsalud

Cuadro 1.

Mantenimiento de los efectos producidos por la rehabilitación pulmonar en pacientes con EPOC: resumen de artículos con mayor peso informativo

\begin{tabular}{|c|c|c|c|}
\hline Autores & $\begin{array}{l}\text { Año de } \\
\text { publicación }\end{array}$ & Persistencia de los efectos & Efectos del entrenamiento \\
\hline Behnke et al. & 2003 & 18 meses & $\begin{array}{l}\text { Tolerancia al ejercicio. CVRS. } \\
\text { Exacerbaciones. }\end{array}$ \\
\hline Engström et al. & 1999 & 12 meses & $\begin{array}{l}\text { Tolerancia al ejercicio. CVRS (no a } \\
\text { largo plazo). Disnea }\end{array}$ \\
\hline Goldstein et al. & 1994 & 6 meses & $\begin{array}{l}\text { Tolerancia al ejercicio. CVRS. } \\
\text { Exacerbaciones. Disnea. }\end{array}$ \\
\hline Griffiths et al. & 2000 & 12 meses & $\begin{array}{l}\text { Tolerancia al ejercicio. CVRS. } \\
\text { Exacerbaciones. Disnea. }\end{array}$ \\
\hline Pitta et al. & 2008 & 6 meses & $\begin{array}{l}\text { Tolerancia al ejercicio. CVRS. } \\
\text { Disnea. }\end{array}$ \\
\hline Puente-Maestu L et al. & 2003 & 13 meses & Tolerancia al ejercicio. \\
\hline Regiane et al. & 2007 & 6 meses & Tolerancia al ejercicio. CVRS. Disnea \\
\hline Ries ALet al. & 2003 & $\begin{array}{l}12 \text { meses mantenimiento fueron } \\
\text { decreciendo más de } 2 \text { años }\end{array}$ & $\begin{array}{llll}\begin{array}{l}\text { Tolerancia al ejercicio. CVRS. } \\
\text { Disnea. }\end{array} & & \\
\end{array}$ \\
\hline Ringbaek et al. & 2010 & 12 meses & Tolerancia al ejercicio. \\
\hline Spencer et al. & 2010 & 12 meses & Tolerancia al ejercicio. CVRS. Disnea \\
\hline Troosters et al. & 2000 & $\begin{array}{l}18 \text { meses con valores } \\
\text { significativos, pero decreciendo a } \\
\text { partir de los } 6 \text { meses }\end{array}$ & $\begin{array}{l}\text { Tolerancia al ejercicio. CVRS. } \\
\text { Disnea. }\end{array}$ \\
\hline
\end{tabular}

Fuente: Elaboración propia.

Se debe rescatar que para mantener los beneficios de la rehabilitación se requieren incluir programas de mantenimiento continuos y cursos de repetición. Continuar con la rehabilitación por un periodo prolongado solo parece tener un pequeño beneficio adicional. Criner et al. (1999) proporcionan en su programa pequeños reciclajes terminada la rehabilitación con efectos positivos.

No está clara la forma que debería adoptar la terapia de mantenimiento o cómo debe aplicarse. Intervenciones mensuales y soporte telefónico parecen mostrar beneficios mientras se aplican, pero su efecto desaparece rápidamente con la suspensión. Ries et al. (1995) proponen un reciclaje y un apoyo o alentamiento telefónico, que funciona pero con muy breve persistencia en el tiempo (Boxall et al., 2005). La repetición de un curso de rehabilitación parece tener la capacidad de reproducir la ganancia a corto plazo, pero no resulta en ventaja a largo plazo.

Un paciente con una rehabilitación pulmonar relativamente corta en el tiempo, ha sido reconocido como piedra angular en el amplio manejo de pacientes con EPOC. La evidencia de 
mejora en la resistencia al ejercicio, la disnea, la capacidad funcional y la calidad de vida sobre la utilización de servicios sanitarios es cada vez mayor.

Finalmente, no existe ninguna norma o regla con respecto a la duración óptima de un programa de rehabilitación pulmonar en EPOC. Desde el punto de vista del paciente, la duración óptima será aquella en la que se produce los efectos máximos del tratamiento, produciendo un aumento significativo en la tolerancia al ejercicio, la disnea y la CVRS.

Más allá de 12 semanas de tratamiento se requiere de cambios complejos de comportamiento para que los pacientes puedan adaptarse, la exposición al tratamiento y la interacción con el personal que proporcionan fortalecimiento y entrenamiento. Además, incluyen la incorporación del ejercicio regular en el estilo de vida del paciente, el uso de técnicas respiratorias, el ritmo y la energía, estrategias de conservación y el uso de medicamentos y equipos de oxígeno suplementario.

Quedan aparte los factores psicosociales y los factores externos que influyen en la duración del programa como el sistema de política de reembolso, el acceso a los programas y el personal de la salud. Como último factor se encuentra la capacidad de cada uno de los pacientes para avanzar hacia metas de tratamiento más exigentes. Los beneficios clínicos de la rehabilitación pulmonar pueden depender tanto del lugar del programa, del contenido y de su duración. El principal problema de un programa de larga duración son las posibles exacerbaciones o enfermedades ajenas al problema, la pérdida de interés o motivación, el transporte, la depresión, el aspecto económico y otras cuestiones de índole personal que rodean la vida de los pacientes.

La duración mínima del entrenamiento físico en la rehabilitación pulmonar no ha sido ampliamente investigada. El entrenamiento ambulatorio con 2 o 3 sesiones semanales durante aproximadamente 4 semanas mostró un beneficio menor que una formación similar durante 7 semanas según los estudios de Green, Singh, Williams y Morgan (2001), quienes compararon dos grupos con similar entrenamiento pulmonar diferenciados únicamente por el tiempo de rehabilitación. Plankeel, McMullen y MacIntyre (2005) plantearon esta misma fórmula con idénticos resultados. Por su parte, Rossi et al. (2005) demuestran en un estudio de 2005 que 20 sesiones de rehabilitación pulmonar global mejoran más los resultados que aplicando 10 sesiones. También se ha demostrado mayor eficacia, según Fuchs-Climent, Le Gallais, Varray, Desplan, Cadopi y Prefaut (1999), que a corto plazo, ya que los programas intensivos de 20 sesiones condensadas en 3-4 semanas mejoran los síntomas y la persistencia de los efectos. En general, se cree que en los programas con mayor rendimiento, los efectos del entrenamiento son más soportables. Salman, Mosier, Beasley y Calkins (2003) y Lacasse et al. (2002) lo muestran en sus estudios en los cuales comparan a dos grupos con distintos rendimientos.

Los pacientes según los ensayos de Ringbaek (2000) y Puente-Maestu et al. (2003) deben realizar ejercicios al menos tres veces por semana con supervisión y regulando las sesiones de ejercicio, esto es necesario para lograr beneficios óptimos fisiológicos (Ringbaek, 2000). Por su parte, Vogiatzis (2002) plantea otra alternativa de entrenamiento supervisado de ejercicios dos veces por semana y una o más sesiones sin supervisión en el hogar, lo cual puede ser aceptado 
Miguel Avenza Jaén

Revista en Ciencias del Movimiento Humano y Salud

URL: http://www.revistas.una.ac.cr/mhsalud

como alternativa, aunque no está claro si esto es tan eficaz. En cambio, Engström (1999) prueba con sesiones supervisadas una vez a la semana, las cuales parecen ser insuficientes.

\section{Conclusiones}

La literatura revisada ha reafirmado la base científica y ha fortalecido la evidencia, la cual demuestra que un programa de entrenamiento rehabilitador pulmonar en pacientes con EPOC mejora los síntomas reduciendo las exacerbaciones.

Se debe considerar el número de personas que forman parte de la rehabilitación. En función de la revisión de literatura realizada, es claro que los programas individualizados son más efectivos que aquellos realizados a nivel colectivo. El trato correcto del terapeuta hacia el paciente es una relación de cercanía y ayuda pero se debe evitar la implicación excesiva por posibles dependencias que dificulten la continuidad del tratamiento en el domicilio de la persona que se encuentra en rehabilitación.

Otra cuestión importante es el lugar de rehabilitación. La principal discusión se ubica en el uso ambulatorio, más caro pero más eficaz a la hora de prolongar los efectos a lo largo del tiempo. Probablemente porque el conjunto de profesionales sanitarios que se implican y sus conocimientos facilitan al paciente su participación. El domicilio es la otra alternativa para llevar a la práctica el tratamiento rehabilitador, nada caro para el sistema socio-sanitario pero ineficaz y falto de seguridad sino se ha tenido un buen adiestramiento o entrenamiento previo. Sí se puede considerar válido cuando lo usamos como complemento al tratamiento ambulatorio y este se revisa con frecuencia por un profesional. El resultado será el aumento del mantenimiento de los efectos conseguidos con la rehabilitación. Quizás la fusión de ambas opciones, bien conjugadas y de forma simultánea, puede ser la fórmula más acertada para no incrementar el gasto sanitario sin perder el efecto tempranamente. Aprovecharnos de estas estrategias de mantenimiento de los efectos puede ayudar a que el paciente se encuentre tratado sin tener que estar supervisado en todo momento por el profesional requerido y sin perder garantías.

La educación o formación del paciente es otro componente necesario y central en un correcto programa rehabilitador pulmonar. No se ha podido identificar un número suficiente de estudios que se centraran solo en la educación. Aunque la evidencia científica es insuficiente, la opinión de expertos apoya la inclusión educativa y las intervenciones psicosociales como componentes de la rehabilitación pulmonar para pacientes con EPOC.

Los principales trastornos psicológicos en la EPOC son la depresión y la ansiedad. Se ha confirmado que existe una alta prevalencia. Por ello, las intervenciones psicosociales serán un factor más a tener en cuenta a la hora de conseguir un beneficio. La evidencia hasta la fecha resulta de incompatibilidad de las intervenciones con los beneficios a corto plazo, no así a largo plazo, que suelen ser beneficiosas. 
Se debe concluir apelando a la falta de un protocolo correcto, efectivo y cerrado en la duración, en el tipo de entrenamiento (aeróbico, interválico o continuo), en el material utilizado (biciergométrica, tapiz rodante, mancuernas, lastres, tiras elástica), en el uso de la fuerza y límites de esta, en la educación y todo lo que conlleva esta, en las intervenciones psicosociales, en el suplemento o no de oxigenoterapia, en la Electroestimulación, la VNI o las posibles estrategias de mantenimiento. Lo único claro y como principal conclusión es que, al no existir una fórmula reconocida para un programa universal que convenga a cualquier sistema, es el cambio de vida del paciente, una mejora en su estilo de vida que conllevé a un cambio.

\section{Recomendaciones}

La revisión bibliográfica efectuada permitió visualizar un campo lleno de oportunidades y desafíos en la rehabilitación pulmonar para mejorarlo y ampliarlo. Se evidencia la necesidad de estudiar y demostrar pruebas más concluyentes sobre la durabilidad de los efectos del entrenamiento. Existen pocos trabajos o ensayos que justifiquen el tiempo concreto de durabilidad de los efectos y qué tipo de entrenamiento concreto y estrategias debemos llevar para aumentar dichos efectos en el tiempo.

También hay necesidad de más estudios sobre resultados psicosociales y su intervención, influencia de los síntomas depresivos en la supervivencia entre los pacientes. Y si estos tienen relación con los otros efectos y su durabilidad en el tiempo.

No ha habido estudios sistemáticos exclusivos de evaluación de la transmisión de la educación, estilos de aprendizaje específicos para cada paciente, la duración de sesiones educativas, la selección de temas, y el uso de refuerzos educativos. Cómo la educación hace mejorar la durabilidad de los efectos por el cambio AVD y buenos hábitos de vida.

Finalmente, se podría investigar sobre el uso de las nuevas tecnologías para poder aprovecharnos de la educación, la vigilancia y el control del paciente con información actual.

Se recomienda realizar investigación futura sobre la duración del tratamiento de rehabilitación pulmonar y estrategias post-rehabilitación para ayudar a los pacientes a mantener la adquisición de los beneficios en un periodo más largo de tiempo.

Por último existen pocos estudios suficientes para determinar la mejora del entrenamiento pulmonar frente al gasto del sistema sanitario en pacientes con EPOC realizando el tratamiento rehabilitador. Todo esto, en un futuro, puede aclararnos más sobre los programas rehabilitadores en pacientes con EPOC.

\section{Referencias bibliográficas}

Barakat S, Michele G, George P, Nicole V, Guy A (2008). Outpatient pulmonary rehabilitation in patients with chronic obstructive pulmonary disease. International Journal of Chronic Obstructive Pulmonary, 3(1),155-62. 
Behnke M,Jörres RA, Kirsten D, Magnussen H. Monaldi (2003). . Clinical benefits of a combined hospital and home-based exercise programme over 18 moths in patients with severe COPD. Archives of Chest Disease, 59(1),44-51.

Boxall AM, Barclay L, Sayers A, Caplan GA (2005) Managing chronic obstructive pulmonary disease in the community. A randomized controlled trial of home-based pulmonary rehabilitation for elderly housebound patients. Journal of Cardiopulm Rehabil, 25(6),378-85.

Brotons B, Pérez JA, Sánchez-Toril F, Soriano S, Hernández J, Belenguer JL (1994). Prevalencia de la enfermedad pulmonar obstructiva crónica y del asma. Estudio transversal. Archivos de Bronconeumología,30, 149-152 http://dx.doi.org/10.1016/S0300-2896(15)31109-1

California Pulmonary Rehabilitation Collaborative Group (2004). Effects of pulmonary rehabilitation on dyspnea, quality of life, and healthcare costs in California. Journal of Cardiopulm Rehabil, 24(1),52-62.

Cambach W, Wagenaar RC, Koelman TW, van Keimpema AR, Kemper HC (1999). The longterm effects of pulmonary rehabilitation in patients with asthma and chronic obstructive pulmonary disease: a research synthesis. Archives of Physical Medicine and Rehabilitation, 80,103-111. http://dx.doi.org/10.1016/S0003-9993(99)90316-7

Casaburi R, Kukafka D, Cooper CB, Witek TJ Jr y Kesten S (2005).. Improvement in exercise tolerance with the combination of tiotropium and pulmonary rehabilitation in patients with COPD. Chest Journal, Mar,127(3),809-817. http://dx.doi.org/10.1378/chest.127.3.809

Criner GJ,Cordova FC, Furukawa S,KuzmaAM, Travaline JM, Leyenson V y O'Brien GM (1999). Prospective randomized trial comparing bilateral lung volume reduction surgery to pulmonary rehabilitation in severe. American Journal of Respiratory and Critical Care Medicine,160(6),2018-2027.

Engström CP, Persson LO, Larsson S, Sullivan M y Scand (1999) Long-term effects of a pulmonary rehabilitation programme in outpatients with chronic obstructive pulmonary disease: a randomized controlled study. Journal of Rehabil Medicine, 31(4),207-13. http:// www.cebp.nl/media/m554.pdf

Finnerty JP, Keeping I, Bullough I, Jones J..(2001).The effectiveness of outpatient pulmonary rehabilitation in chronic lung disease: a randomized controlled trial. Chest Journal, 119 (6), 1705-10.

Foglio K, Bianchi L, Bruletti G, Battista L, Pagani M, Ambrosino N (1999). Long-term effectiveness of pulmonary rehabilitation in patients with chronic airway obstruction. European Respiratory Journal, 13,125-132. http://dx.doi.org/10.1183/09031936.99.13112599 
Fuchs-Climent D, Le Gallais D, Varray A, Desplan J, Cadopi M y Prefaut C (1999). Quality of life and exercise tolerance in chronic obstructive pulmonary disease: effects of a short and intensive inpatient rehabilitation program. American Journal of Physical Medicine \& Rehabilitation.78:330-335. http://dx.doi.org/10.1097/00002060-199907000-00007

Global Initiative for Chronic Obstructive Lung Disease (GOLD). (2006). Estrategia Global para Diagnóstico, tratamiento y prevención de la enfermedad pulmonar obstructiva crónica, S.d: Medical Communications Resources, Inc.

Gold J y Shadlen M (2007). The neural basis of decision making. Annual Review of Neuroscience, 30,535-574. http://dx.doi.org/10.1146/annurev.neuro.29.051605.113038

Goldstein RS, Gort EH, Stubbing D, Avendano MA, Guyatt GH (1994). Randomised controlled trial of respiratory rehabilitation. The Lancet Journal, 344,1394-1397. http://dx.doi. org/10.1016/S0140-6736(94)90568-1

Green RH, Singh SJ, Williams J,Morgan MD (2001). A randomised controlled trial of four weeks versus seven weeks of pulmonary rehabilitation in chronic obstructive pulmonary disease. Thorax Journal, 56,143-145. http://dx.doi.org/10.1136/thorax.56.2.143

Griffiths TL, Burr ML, Campbell IA, Lewis-Jenkins V, Mullins J, Shiels K...y Tunbridge J (2000).Results at 1 year of outpatient multidisciplinary pulmonary rehabilitation: a randomised controlled trial. The Lancet Journal, 355(9201),362-368.

Guell R, Casan P, Belda J, Sangenis M, Morante F, Guyatt GH, Sanchis J (2000). Long-term effects of outpatient rehabilitation of COPD: a randomized trial. Chest Journal,117,976-983. http://dx.doi.org/10.1378/chest.117.4.976

Güell R, Resqueti V, Sangenis M, Morante F, Martorell B, Casan P y Guyatt GH. (2006) Impact of pulmonary rehabilitation on psychosocial morbidity in patients with severe COPD. Chest Journal,129(4),899-904

Heppner PS, Morgan C, Kaplan RM, Ries AL (2006). Regular walking and long-term maintenance of outcomes after pulmonary rehabilitation. Journal of Cardiopulm Rehabil. 26 (1),44-53. http://dx.doi.org/10.1097/00008483-200601000-00010

Karapolat H, Atasever A, Atamaz F, Kirazli Y, Elmas F, Erdinç E. Lung. (2007) Do the benefits gained using a short-term pulmonary rehabilitation program remain in COPD patients after participation. Lung Journal, 185 (4),221-5. http://www.ncbi.nlm.nih.gov/pubmed/17487535

Lacasse Y, Brosseau L, Milne S, Martin S, Wong E, Guyatt GH y Goldstein RS (2002). Pulmonary rehabilitation for chronic obstructive pulmonary disease. Cochrane Database and Systematic Rev, 3:CD003793. http://www.ncbi.nlm.nih.gov/pubmed/17054186 
Nici L, Donner C, Wouters E, Zuwallack R, Ambrosino N, Bourbeau J... y ATS/ERS Pulmonary Rehabilitation Writing Committee (2006). American Thoracic Society/ European Respiratory Society statement on pulmonary rehabilitation. American Journal of Respiratory and Critical Care Medicine,173,1390-1413

Peña VS, Miravitlles M, Gabriel R, Jiménez-Ruiz CA, Villasante C, Masa JF (2000) Geographic Variations in Prevalence and Underdiagnosis of COPD. Results of the IBERPOC Multicentre Epidemiological Study. Chest Journal,118 (4), 981-989. http:// dx.doi.org/10.1378/chest.118.4.981

Pitta F, Troosters T, Probst VS, Langer D, Decramer M, Gosselink R (2008) Are patients with COPD more active alter pulmonary rehabilitation. Chest Journal,134(2),273-80.http:// doi: $\underline{10.1378 / \text { chest.07-2655 }}$

Plankeel JF, McMullen B y MacIntyre NR (2005). Exercise outcomes after pulmonary rehabilitation depend on the initial mechanisms of exercise limitation among non-oxygen-dependent COPD patients. Chest Journal, 127:110-116. http://dx.doi.org/10.1378/chest.127.1.110

Pride NB. (1990 ). Chronic obstructive pulmonary disease. Epidemiology, etiology and natural history. Respiratory Medicine (Sd.). London : Baillière Tindall.

Puente-Maestu L, SantaCruz A, Vargas T, Martínez-Abad Y y ·Whipp B.J (2003). Effects of Training on the Tolerance to High-Intensity Exercise in Patients with Severe COPD. Respiration,70(4),367-370. http://dx.doi.org/10.1159/000072899

Puhan MA, Scharplatz M, Troosters T, Steurer J. (2005) Respiratory rehabilitation after acute exacerbation of COPD may reduce risk for readmision and mortality: a systematic review. Respiratory Research.,6,54. http://dx.doi.org/10.1186/1465-9921-6-54

Regiane Resqueti V, Gorostiza A, Gáldiz JB, López de Santa María E, Casan Clará P, Güell Rous R (2007). Benefits of a home-based pulmonary rehabilitation program for patients with severe chronic obstructive pulmonary disease. Archivos de Bronconeumología,43(11):599-604.

Ries AL, Kaplan RM, Limberg TM, Prewitt LM (1995). Effects of pulmonary rehabilitation on physiologic and psychosocial outcomes in patients with chronic obstructive pulmonary disease. Annals of Internal Medicine: Journal, 122,823-832. http://dx.doi.org/10.7326/00034819-122-11-199506010-00003

Ries AL, Kaplan RM, Myers R, Prewitt LM (2003); Maintenance alter pulmonary rehabilitation in chronic lung disease. Lung Journal, 181(2),67-78. http://dx.doi.org/10.1007/s00408-003-1007-0

Ries L; Bauldoff S, Carlin W; Casaburi R;. Emery C ; Mahler D... Herrerias C. (2007). Pulmonary rehabilitation: joint ACCP/AACVPR evidence-based guidelines. Chest Journal, 112(5), 1363-1396. doi:10.1378/chest.06-2418/ http://www.ncbi.nlm.nih.gov/pubmed/17494825 
Ringbaek TJ, Broendum E, Hemmingsen L, Lybeck K, Nielsen D,Andersen C y Lange P (2000) Rehabilitation of patients with chronic obstructive pulmonary disease: exercise twice a week is not sufficient! Respiratory Medicine, 94,150-154. http://dx.doi.org/10.1053/ rmed.1999.0704

Rossi G, Florini F, Romagnoli M, Bellatone T, Lucic S, Lugli D, Clini E. (2005) Length and clinical effectiveness of pulmonary rehabilitation in outpatients with chronic airway obstruction. Chest Journal,127,105-109. http://dx.doi.org/10.1378/chest.127.1.105

Salman GF, Mosier MC, Beasley BW y Calkins DR (2003). Rehabilitation for patients with chronic obstructive pulmonary disease. Journal of General Internal Medicine,18,213-221. http://dx.doi.org/10.1046/j.1525-1497.2003.20221.x

Sobradillo V, Miravitlles M, Jiménez CA, Gabriel R, Viejo JL, Masa JF, Fernández-Fau L y Villasant C (1999). Estudio IBERPOC en España: prevalencia de síntomas respiratorios habituales y de limitación crónica al flujo aéreo. Archivos de Bronconeumología, 35,159166. http://dx.doi.org/10.1016/S0300-2896(15)30272-6

Sociedad Española de Neumología y Cirugía Toráxica y Asociación Latinoamericana de Tórax (SEPAR y ALLAT-ULASTER). (2009) Guía de práctica clínica de diagnóstico y tratamiento de la EPOC.

Spencer LM, Alison JA, McKeough ZJ (2010) Maintaining benefits following pulmonary rehabilitation..European Respiratory Journal, 35(3),571-7. http:// doi: $\underline{10.1183 / 09031936.00073609}$

Strijbos JH, Postma DS, van Altena R, Gimeno F, Koeter GH (1996). A comparison between an outpatient hospital-based pulmonary rehabilitation program and a home-care pulmonary rehabilitation program in patients with COPD: a follow-up of 18 moths. Chest Journal, 109,366-372. http://dx.doi.org/10.1378/chest.109.2.366

Toshima MT, Kaplan RM, Ries AL (1990) Experimental evaluation of rehabilitation in chronic obstructive pulmonary disease: short-term effects on exercise endurance and health status. . Health Psychol, 9(3),237-52. http://dx.doi.org/10.1037/0278-6133.9.3.237

Troosters T, Gosselink R, Decramer M (2000) Short- and long-term effects of outpatient rehabilitation in patients with chronic obstructive pulmonary disease: a randomized trial. The American Journal of Medicine, 109,207-212. http://dx.doi.org/10.1016/S0002$\underline{9343(00) 00472-1}$

Vogiatzis I, Nanas S y Roussos C (2000) Interval training as an alternative modality to continuous exercise in patients with COPD. European Respiratory Journal, 20,12-19. http://dx.doi.org/10.1183/09031936.02.01152001 
Wedzicha JA, Bestall JC, Garrod R, Garnham R, Paul EA, Jones PW (1998). Randomized controlled trial of pulmonary rehabilitation in severe chronic obstructive pulmonary disease patients, stratified with the MRC dyspnoea scale. European Respiratory Journal, 12,363-369. http://dx.doi.org/10.1183/09031936.98.12020363

Wijkstra PJ, Ten Vergert EM, van Altena R, Otten V, Kraan J, Postma DS y Koëter GH (1995). Long term benefits of rehabilitation at home on quality of life and exercise tolerance in patients with chronic obstructive pulmonary disease. Thorax Journal, 50(8),824-8.

World Health Organization. (2008). Global Burden of Disease: 2004 update. Sd: WHO. http:// www.who.int/healthinfo/global_burden_disease/GBD_report_2004update full.pdf

Recepción: 02 de febrero del 2015.

Corrección: 23 de junio del 2015.

Aceptación: 19 de agosto del 2015

Publicación: 26 de agosto del 2015.

1 Agradecimientos: Al Dr. Roberto Ravinovich, Neumólogo e investigador del Edinburgh University Charitable Status por el asesoramiento prestado en la revisión bibliográfica y las sugerencias técnicas proporcionadas. Extensivo el agradecimiento a la Biblioteca de Medicina de la Universidad Autónoma de Madrid por dar acceso a todas las bases de datos.

2 El autor es fisioterapeuta del Hospital Carlos III Madrid, España 